\title{
Using augmented reality devices for remote support in manufacturing: A case study and analysis
}

\author{
Buń, P. ${ }^{a,}{ }^{*}$, Grajewski, D. ${ }^{a}$, Górski, F. ${ }^{\text {a }}$ \\ aPoznan University of Technology, Poznań, Poland
}

\begin{abstract}
A B S T R A C T
Industry 4.0 forces increased digitization, production flexibility, improvement of employee competences and integration of employees and IT systems within an enterprise. To this end, state-of-the-art systems and IT solutions, such as the Virtual Reality (VR) and Augmented Reality (AR), are implemented. New systems must be integrated with the existing IT architecture, and their implementation forces the enterprise to provide network access with sufficient bandwidth to fully benefit from the capabilities of new technologies. The paper discusses the practical application of modern AR solutions in the industry, with a special focus on remote support for maintenance operations and training of production employees. Two experiments aimed determining the impact of various environmental conditions on the possibility of using the AR Remote Support are described. Basing on those experiments it is possible to determine the environmental conditions required to use HoloLens 2 AR goggles in two dedicated remote support applications.
\end{abstract}

\author{
ARTICLE INFO \\ Keywords: \\ Smart manufacturing; \\ Industry 4.0; \\ Remote support; \\ Augmented reality (AR); \\ Virtual reality; \\ HoloLens 2; \\ Ambient noise; \\ Wi-Fi networks \\ *Corresponding author: \\ pawel.bun@put.poznan.pl \\ (Buń, P.) \\ Article history: \\ Received 4 October 2021 \\ Revised 10 December 2021 \\ Accepted 11 December 2021

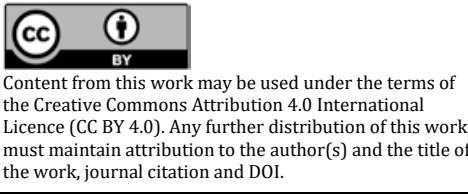

\section{References}

[1] Sari, T., Güleș, H.K., Yiğitol, B. (2020). Awareness and readiness of Industry 4.0: The case of Turkish manufacturing industry, Advances in Production Engineering \& Management, Vol. 15, No.1, 57-68, doi: 10.14743/apem 2020.1.349.

[2] Oesterreich, T.D., Teuteberg, F. (2016). Understanding the implications of digitisation and automation in the context of industry 4.0: A triangulation approach and elements of a research agenda for the construction industry, Computers in Industry, Vol. 83, 121-139, doi: 10.1016/j.compind.2016.09.006.

[3] Romero, D., Stahre, J., Taisch, M. (2020). The Operator 4.0: Towards socially sustainable factories of the future, Computers \& Industrial Engineering, Vol. 139, Article No. 106128, doi: 10.1016/j.cie.2019.106128.

[4] Żywicki, K., Zawadzki, P., Górski, F. (2018). Virtual reality production training system in the scope of intelli-gent factory, Advances in Intelligent Systems and Computing, Vol. 637, 450-458, doi: 10.1007/978-3-319-64465-3 43.

[5] Marino, E., Barbieri, L., Colacino, B., Fleri, A.K., Bruno, F. (2021). An Augmented Reality inspection tool to support workers in Industry 4.0 environments, Computers in Industry, Vol. 127, Article No. 103412, doi: 10.1016/i.compind.2021.103412.

[6] Urbas, U., Ariansyah, D., Erkoyuncu, J.A., Vukašinović, N. (2021). Augmented reality aided inspection of gears, Tehnički Vjesnik - Technical Gazette, Vol. 28, No. 3, 1032-1037, doi: 10.17559/TV-20200728151912. 
[7] Uva, A.E., Gattullo, M., Manghisi, V.M., Spagnulo, D., Cascella, G.L., Fiorentino, M. (2018). Evaluating the effectiveness of spatial augmented reality in smart manufacturing: A solution for manual working stations, The International Journal of Advanced Manufacturing Technology, Vol. 94, 509-521, doi: 10.1007/s00170-017-0846-4.

[8] Castellanos, M.J., Navarro-Newball, A.A. (2019). Prototyping an augmented reality maintenance and repairing system for a deep well vertical turbine pump, In: 2019 International Conference on Electronics, Communications and Computers (CONIELECOMP), 36-40, doi: 10.1109/CONIELECOMP.2019.8673254.

[9] Bottani, E., Vignali, G. (2019). Augmented reality technology in the manufacturing industry: A review of the last decade, IISE Transactions, Vol. 51, No. 3, 284-310, doi: 10.1080/24725854.2018.1493244.

[10] Kulkov, I., Berggren, B., Hellström, M., Wikström, K. (2021). Navigating uncharted waters: Designing business models for virtual and augmented reality companies in the medical industry, Journal of Engineering and Technology Management, Vol. 59, Article No. 101614, doi: 10.1016/j.jengtecman.2021.101614.

[11] Palmarini, R., Erkoyuncu, J.A., Roy, R., Torabmostaedi, H. (2018). A systematic review of augmented reality applications in maintenance, Robotics and Computer-Integrated Manufacturing, Vol. 49, 215-228, doi: 10.1016/j.rcim. 2017.06.002.

[12] Vujica Herzog, N., Buchmeister, B., Beharic, A., Gajsek, B. (2018). Visual and optometric issues with smart glasses in Industry 4.0 working environment, Advances in Production Engineering \& Management, Vol. 13, No. 4, 417 428, doi: 10.14743/apem2018.4.300.

[13] Ellingsen, O., Aasland, K.E. (2019). Digitalizing the maritime industry: A case study of technology acquisition and enabling advanced manufacturing technology, Journal of Engineering and Technology Management, Vol. 54, 12-27, doi: 10.1016/j.jengtecman.2019.06.001.

[14] Ludwig, T., Stickel, O., Tolmie, P., Sellmer, M. (2021). shARe-IT: Ad hoc remote troubleshooting through augmented reality, Computer Supported Cooperative Work (CSCW), Vol. 30, 119-167, doi: 10.1007/s10606-021-09393-5.

[15] Obermair, F., Althaler, J., Seiler, U., Zeilinger, P., Lechner, A., Pfaffeneder, L., Richter M., Wolfartsberger, J. (2020). Maintenance with augmented reality remote support in comparison to paper-based instructions: Experiment and analysis, In: 2020 IEEE 7th International Conference on Industrial Engineering and Applications (ICIEA), 942-947, doi: 10.1109/ICIEA49774.2020.9102078.

[16] Chang, Y.S., Nuernberger, B., Luan, B., Höllerer, T. (2017). Evaluating gesture-based augmented reality annotation, In: 2017 IEEE Symposium on 3D User Interfaces (3DUI), 182-185, doi: 10.1109/3DUI.2017.7893337.

[17] Egger, J., Masood, T. (2020). Augmented reality in support of intelligent manufacturing - A systematic literature review, Computers \& Industrial Engineering, Vol. 140, Article No. 106195, doi: 10.1016/i.cie.2019.106195.

[18] Syberfeldt, A., Holm, M., Danielsson, O., Wang, L., Brewster, R.L. (2016). Support systems on the industrial shopfloors of the future - Operators' perspective on augmented reality, Procedia CIRP, Vol. 44, 108-113, doi: 10.1016/ j.procir.2016.02.017.

[19] AR4vision. Benefits of Augment Reality, from https://ar4vision.pl/\#korzysci, accessed September 7, 2021.

[20] Ojer, M., Alvarez, H., Serrano, I., Saiz, F.A., Barandiaran, I., Aguinaga, D., Querejeta, L., Alejandro, D. (2020). Projection-based augmented reality assistance for manual electronic component assembly processes, Applied Sciences, Vol 10, No. 3, 796, doi: 10.3390/app10030796.

[21] Blattgerste, J., Renner, P., Strenge, B., Pfeiffer, T. (2018). In-situ instructions exceed side-by-side instructions in augmented reality assisted assembly, In: Proceedings of the 11th PErvasive Technologies Related to Assistive Environments Conference, 133-140, doi: 10.1145/3197768.3197778.

[22] Kranzer, S., Prill, D., Aghajanpour, D., Merz, R., Strasser, R., Mayr, R., Zoerrer, H., Plasch, M., Steringer, R. (2017). An intelligent maintenance planning framework prototype for production systems, In: 2017 IEEE International Conference on Industrial Technology (ICIT), doi: 10.1109/ICIT.2017.7915520.

[23] Del Amo, I.F., Erkoyuncu, J.A., Roy, R., Wilding, S. (2018). Augmented reality in maintenance: An information-centred design framework, Procedia Manufacturing, Vol. 19, 148-155, doi: 10.1016/j.promfg.2018.01.021.

[24] Palmarini, R., Erkoyuncu, J.A., Roy, R., Torabmostaedi, H. (2018). A systematic review of augmented reality applications in maintenance, Robotics and Computer-Integrated Manufacturing, Vol. 49, 215-228, doi: 10.1016/i.rcim. 2017.06.002.

[25] Antonelli, D., Astanin, S. (2015). Enhancing the quality of manual spot welding through augmented reality assisted guidance, Procedia CIRP, Vol. 33, 556-561, doi: 10.1016/j.procir.2015.06.076.

[26] Ramakrishna, P., Hassan, E., Hebbalaguppe, R., Sharma, M., Gupta, G., Vig, L., Sharma, G., Shroff, G. (2016). An AR inspection framework: Feasibility study with multiple ar devices. In: 2016 IEEE International Symposium on Mixed and Augmented Reality (ISMAR-Adjunct), 221-226, doi: 10.1109/ISMAR-Adjunct.2016.0080.

[27] Galkin, M., Auer, S., Scerri, S. (2016). Enterprise knowledge graphs: A backbone of linked enterprise data, In: 2016 IEEE/WIC/ACM International Conference on Web Intelligence (WI), 497-502, doi: 10.1109/WI.2016.0083.

[28] Fraga-Lamas, P., Fernandez-Carames, T.M., Blanco-Novoa, O., Vilar-Montesinos, M.A. (2018). A review on industrial augmented reality systems for the industry 4.0 shipyard, IEEE Access, Vol. 6, 13358-13375, doi: 10.1109/ACCESS.2018.2808326.

[29] Sabri, Y., Micheli, G.J.L., Nuur, C. (2018). Exploring the impact of innovation implementation on supply chain configuration, Journal of Engineering and Technology Management, Vol. 49, 60-75, doi: 10.1016/i.jengtecman. 2018.06.001.

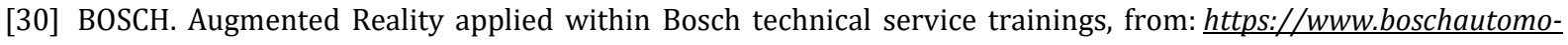
tiveservicesolutions.com/examples-augmented-reality-applications, accessed August 30, 2021.

[31] Parvez, I., Rahmati, A., Guvenc, I., Sarwat, A.I., Dai, H. (2018). A survey on low latency towards 5G: RAN, core network and caching solutions. IEEE Communications Surveys \& Tutorials, Vol. 20, No. 4, 3098-3130 doi: 10.1109/COMST.2018.2841349. 
[32] Siriwardhana, Y., Porambage, P., Liyanage, M., Ylianttila, M. (2021). A survey on mobile augmented reality with 5G mobile edge computing: Architectures, applications and technical aspects, Communications Surveys \& Tutorials, Vol. 23, No. 2, 1160-1192, doi: 10.1109/COMST.2021.3061981.

[33] Microsoft. HoloLens2 specification, from https://docs.microsoft.com/en-us/hololens/hololens2-hardware, accessed August 30, 2021.

[34] Apzumi. Apzumi Spatial, from https://spatial.apzumi.com/, accessed March 30, 2021.

[35] Microsoft. Technical requirements for deploying and using Dynamics 365 Remote Assist, from https://docs.microsoft.com/en-us/dynamics365/mixed-reality/remote-assist/requirements, accessed March 22, 2021.

[36] Microsoft. Prepare your organization's network for Microsoft Teams, from https://docs.microsoft.com/en-us/MicrosoftTeams/prepare-network\#best-practice-monitor-your-network-using-cqd-and-call-analytics, accessed August 22, 2021.

[37] Batalla, J.M. (2020). On analyzing video transmission over wireless Wi-Fi and 5G C-band in harsh IIoT environments, IEEE Access, Vol. 8, 118534-118541, doi: 10.1109/ACCESS.2020.3005641. 


\section{APEM}

\section{Uporaba naprav za razširjeno resničnost za podporo na daljavo v proizvodnji: Študija primera in analiza}

Buń, P. ${ }^{a}{ }^{*}$, Grajewski, D. ${ }^{a}$, Górski, F. ${ }^{a}$

aPoznan University of Technology, Poznań, Poland

\section{POVZETEK}

Industrija $4.0 \mathrm{v}$ podjetja prinaša večjo digitalizacijo, fleksibilnost proizvodnje, izboljšanje kompetenc zaposlenih ter integracijo zaposlenih in IT sistemov. V ta namen podjetja vpeljujejo najsodobnejše sisteme in IT rešitve, kot sta navidezna resničnost (VR) in razširjena resničnost (AR). Novi sistemi morajo biti integrirani v obstoječo IT arhitekturo, njihova vpeljava pa od podjetij zahteva zagotovitev dostopa do omrežja z zadostno pasovno širino. Tako lahko podjetja v celoti izkoristijo zmogljivosti novih tehnologij. Prispevek obravnava praktično uporabo sodobnih AR rešitev v industriji, s posebnim poudarkom na podpori na daljavo namenjeni vzdrževanju in usposabljanju zaposlenih $\mathrm{v}$ proizvodnji. Opisana sta dva poskusa, namenjena ugotavljanju vpliva različnih okoljskih pogojev na možnost uporabe podpore na daljavo z AR. Na podlagi teh poskusov je mogoče določiti okoljske pogoje, potrebne za uporabo očal HoloLens 2 AR v dveh namenskih aplikacijah za oddaljeno podporo.

\section{PODATKI O ČLANKU}

Ključne besede:

Pametna proizvodnja;

Industrija 4.0;

Podpora na daljavo;

Razširjena resničnost (AR);

Navidezna resničnost;

HoloLens 2;

Šum okolice;

Omrežja Wi-Fi

*Kontaktna oseba: pawel.bun@put.poznan.pl

(Buń, P.)

Zgodovina članka:

Prejet 4. oktobra 2021

Popravljen 10. decembra 2021

Sprejet 11. decembra 2021

\section{(i)}

Content from this work may be used under the terms of he Creative Commons Attip Licence (CC BY 4.0). Any further distribution of this wo must maintain attribution to the author(s) and the title of the work, journal citation and DOI. 